\title{
Expression Vectors for Human-mouse Chimeric Antibodies
}

\author{
Hua Xiong, Yuliang Ran ${ }^{\dagger}$, Jinliang Xing, Xiangmin Yang, Yu Li and Zhinan Chen* \\ Cell Engineering Research Center, State Key Laboratory of Cancer Biology, Fourth Military Medical University, \\ Xi' an City, 710032, China \\ 'Department of Cell and Molecular Laboratory, Cancer Institute (Hospital), Chinese Academy of Medical Sciences and \\ Peking Union Medical College, Beijing City, 100021, China
}

Received 10 March 2005, Accepted 15 March 2005

\begin{abstract}
The production of recombinant antibodies has been generally recognized as time-consuming and labor-intensive. The aim of our study is to construct mammalian expression vectors containing the cDNA encoding the human constant regions and murine variable regions to massively and costeffectively produce full-length chimeric antibodies. Unique restriction sites flanking the Ig variable region were designed to allow for the replacement of variable regions generated by PCR. Western blot analysis of the chimeric antibodies revealed that the expressed products were of the predicted size, structure and specificity. The usefulness of the vectors was confirmed by construction of humanmouse chimeric antibody-HCAb which secretes murine antibody against the human colorectal cancer. Selected in medium containing gradually increasing methotrexate (MTX), clones with increased expression of the product gene can be efficiently generated. The secretion of recombinant chimeric antibody-HCAb yielded $30 \mathrm{pg} \mathrm{cell}^{-1}$ day $^{-1}$ at $10^{-6}$ M MTX. With this high-level expression from pools, the convenient and rapid production of over 100 milligram amounts per liter of recombinant antibodies may be achieved, which indicates the significant roles of pYR-GCEVH and pYR-GCEVL in the production of chimeric antibodies.
\end{abstract}

Keywords: Antibody engineering, Chimeric antibody, Chinese hamster ovary cells, Eucaryotic expression, Expression vector

\section{Introduction}

The derivation of monoclonal antibodies using classic techniques has yielded many antibodies playing significant roles in both

\footnotetext{
* To whom correspondence should be addressed.

Tel: 86-29-83293906; Fax: 86-29-83293906

E-mail: chcerc3@fmmu.edu.cn
}

research and diagnosis (Persic et al., 1997). However, their inherent immunogenicity in patients had hindered their longterm administration in immunosuppressive therapy (Waldmann, 1991). Chimeric or humanized antibodies, which are largely human in composition, have been generated by recombinant DNA techniques (Liu et al., 1987; Burton, 1991; Harris et al., 1993). Recombinant antibodies have been increasingly used as therapeutics for a wide variety of diseases because their immunogenicity may be reduced by employing chimeric antibodies, in which the variable regions of murine antibodies are attached to human constant regions (Morrison, 1985). Thus, establishing high level expression system for chimeric antibodies is very significant.

For high level expression of recombinant antibodies, one of the most widely used mammalian expression systems is the gene amplification procedure offered by the use of dihydrofolate reductase-deficient Chinese hamster ovary cells ( $\left.\mathrm{dhfr}^{-} \mathrm{CHO}\right)$. The advantage of this system lies in its ability to amplify genes linked to DHFR, which leads to enhanced levels of protein expression. This system is based on DHFR gene coding for DHFR enzyme that catalyzes the conversion of dihydrofolate to tetrahydrofolate. MTX binds to DHFR enzyme, but dhfr ${ }^{-} \mathrm{CHO}$ cells, which take up an expression vector containing the DHFR gene, can develop resistance to it by amplifying the DHFR gene. Because the amplification unit is much larger $(100$ to $3000 \mathrm{~kb}$ ) than the size of the DHFR gene, the specific gene of interest, which is colinked to the DHFR gene in the same expression vector or adjacently resides in the host chromosome, can be coamplified (Kaufman et al., 1983).

Most vectors used for antibody expression in mammalian cells have evolved from the pSV2 series (Gorman et al., 1991). However, such vectors have not been optimized for chimeric antibody production. We present here new universal expression vectors that can be used to generate stable clones that consistently express high levels of secreted recombinant antibodies. We have tested the expression vectors using human-mouse chimeric antibody-HCAb, whose V (variable) 
gene was obtained from hybridoma cells using RT-PCR. VH (variable region gene of the heavy chain) and VL (variable region gene of the light chain) were inserted into expression vector $p Y R-G C E V H$ and $p Y R-G C E V L$, respectively. When subjected to successive rounds of selection in medium containing stepwise increments of MTX concentration, recombinant $\mathrm{CHO}$ cells with $\mathrm{HCAb}$ antibody productivity as high as $30 \mathrm{pg}$ cell $^{-1}$ day $^{-1}$ were obtained. We found that the expression vectors can directly and highly express a variety of recombinant antibodies from pools of stable transfectants.

\section{Materials and Methods}

Construction of the heavy chain expression vector pYRGCEVH The expression vector $p Y R-G C E V H$ was derived from the vector pSV2-dhfr (ATCC, USA) and pcDNA3 (Invitrogen, San Diego, USA).We chose pSV2-dhfr as framework, digested it with SphI endonuclease and isolated the larger fragment by gel electrophoresis. Thus, we deleted the $72 \mathrm{bp}$ repetitive sequences of enhanser in SV40 promoter, and obtained the recombinant vector pYR-SV2-rdhfr. Subsequently, the phCMV-MCS-BGH polyA fragment from pcDNA3 was excised and cloned into pYR-SV2rdhfr by BamHI and EcoRI to generate pYR-GSEVH. The expression vector $\mathrm{pYR}-\mathrm{GCEVH}$ was made from $\mathrm{pYR}-\mathrm{GSEVH}$ by insertion of human constant region $(\mathrm{C} \gamma 1$ or $\mathrm{C} \gamma 2$ or $\mathrm{C} \gamma 3$ or $\mathrm{C} \gamma 4)$ into $\mathrm{XhoI}$ site. Therefore, any variable region genes can be introduced into pYR-GCEVH by BamHI and NotI (Fig. 1).

Construction of the light chain expression vector pYR-GCEVL The Neo gene from pSV2-neo (ATCC, USA) was excised by HindIII and BamHI and cloned into pYR-SV2-rdhfr to obtain pYRSV2-rneo, whose SV40 promoter was also weakened, for deletion of $72 \mathrm{bp}$ repetitive sequences like pYR-SV2-rdhfr. And then, excision of the phCMV-MCS-BGH polyA fragment and cloning into pYR-SV2-rneo was performed to generate pYR-GSEVL. The plasmid $p$ YR-GCEVL was obtained by insertion of human constant region $(\mathrm{C} \lambda$ or $\mathrm{C \kappa})$ (Fig. 2).

Construction of expression vectors for Anti-human Colorectal cancer Antibody (HCAb) HCAb was recently developed in our laboratory (Yang et al., 2004), whose light and heavy chain variable region genes were obtained by RT-PCR, and cloned into plasmids by BamHI and NotI.

Cell culture, vectors transfection and selection COS-7 cells (ATCC, USA) were employed for transient antibody expression using LipofectAMINE ${ }^{\mathrm{TM}}$ (GibcoBRL, Life Technologies, USA) transfection reagent according to the manufacturer's description.

For stable transfection, $\mathrm{dhfr}^{-} \mathrm{CHO}$ (ATCC, USA) cells were chosen. Prior to transfection, $\mathrm{dhfr}^{-} \mathrm{CHO}$ cells were maintained in DMEM (Hyclone, Logan, USA) medium supplemented with $10 \%$ (V/V) FCS (GIBCO, Life Technologies, USA), L-glutamine (2 mM), glycine $10 \mu \mathrm{g} \mathrm{ml}^{-1}$, hypoxanthine $15 \mu \mathrm{g} \mathrm{ml}^{-1}$ and thymidine $5 \mu \mathrm{g} \mathrm{ml}^{-1}$ (GHT). Then, samples of $20 \mu \mathrm{g}$ DNA of each expression vector were cotransfected into $10^{6} \mathrm{dhfr}^{-} \mathrm{CHO}$ cells using LipofectAMINE ${ }^{\mathrm{TM}}$ transfection reagent following the manufacturer's instruction. After transfected, cells were cultured in nonselective medium for 3 days, then fed with GHT free DMEM medium that also contained $200 \mu \mathrm{g}$ $\mathrm{ml}^{-1}$ of G418 (GIBCO BRL) to select for DHFR ${ }^{+}$and $\mathrm{Neo}^{+}$clones. After 3 weeks, selected clones were subcloned in 96-well microtiter plates. The culture supernatant was tested for the assembled antibody production by ELISA. And then, the transfected CHO clones were subjected to amplification (stepwise increments in MTX (Sigma, St. Louis, USA) level, such as $3 \times 10^{-8}, 10^{-7}, 10^{-6} \mathrm{M}$ ) and subcloning at each level.

ELISA-assay ELISA analysis was used to assess the productivity of the secreted HCAb. The microtiter plate wells were coated overnight at $4^{\circ} \mathrm{C}$ with $10 \mathrm{mg} \mathrm{L}^{-1}$ of the goat anti-human kappa light chain (Bound and free) antibody (Sigma). Plates were blocked with $1 \% \mathrm{BSA}$ in PBS for at least $2 \mathrm{~h}$ at $37^{\circ} \mathrm{C}$ followed by washing three times with PBS- $0.1 \%$ Tween 20 before culture supernatant was added overnight at $4^{\circ} \mathrm{C}$. After extensive washing with PBS-Tween, goat anti-human $\operatorname{IgG}$ ( $\mathrm{Fc}$ specific)-peroxidase antibody (Sigma) was added at the appropriate dilution and incubated for $2 \mathrm{~h}$ at $37^{\circ} \mathrm{C}$. Further washes were followed by the addition of TMB (3,3',5,5'tetramethylbenzidine) for $15 \mathrm{~min}$ at room temperature and $2 \mathrm{M}$ sulphuric acid was used to stop the assay. Then, OD450 was measured immediately.

Ig quantitation was examined by Elisa using purified human IgG as a standard. The supernatant harvested from $10^{6}$ cells, cultured for $24 \mathrm{~h}$, was assayed to determine the level of production of chimeric antibody HCAb by ELISA (see above). The amount of the antibody was assessed by comparison to a standard curve.

Western blot analysis of recombinant Ig molecules Standard procedures were used to analyse the weight of the recombinant Ig molecules. IgG, HCAb from $20 \mathrm{ml}$ of culture supernatants was purified using a protein-A column (Pierce, Rockford, USA), concentrated using Centricon filtration (Millipore, Bedford, MA) and then subjected to SDS-PAGE under reducing conditions (Laemmli, 1970). Finally, the molecular weight of the individual Ig chains was estimated by western blot using specific peroxidase conjugated anti-human gamma or kappa antibody.

\section{Result}

Construction of Ig light chain and heavy chain expression vectors Two vectors, $p Y R-G C E V H$ for Ig heavy chain and pYR-GCEVL for Ig light chain, were constructed (Fig. 1, 2). The vectors contain BamHI and NotI endonuclease restriction sites flanking the rearranged $\mathrm{V}$ region cDNA generated by PCR. These two vectors contain the hCMV promoter, BGH polyadenylation site, $\mathrm{C}$ region, and origin of replication. As pYR-GCEVH contains the weakened DHFR gene, while pYR-GCEVL contains the Neo gene, co-transfection of the two plasmids into mammalian cells warrants the production of functional, complete Ig molecules of desired antigen binding specificity.

Chimeric antibody production by transfected cells The $\mathrm{dhfr}^{-} \mathrm{CHO}$ cells were transfected with pYR-GCEVH and 

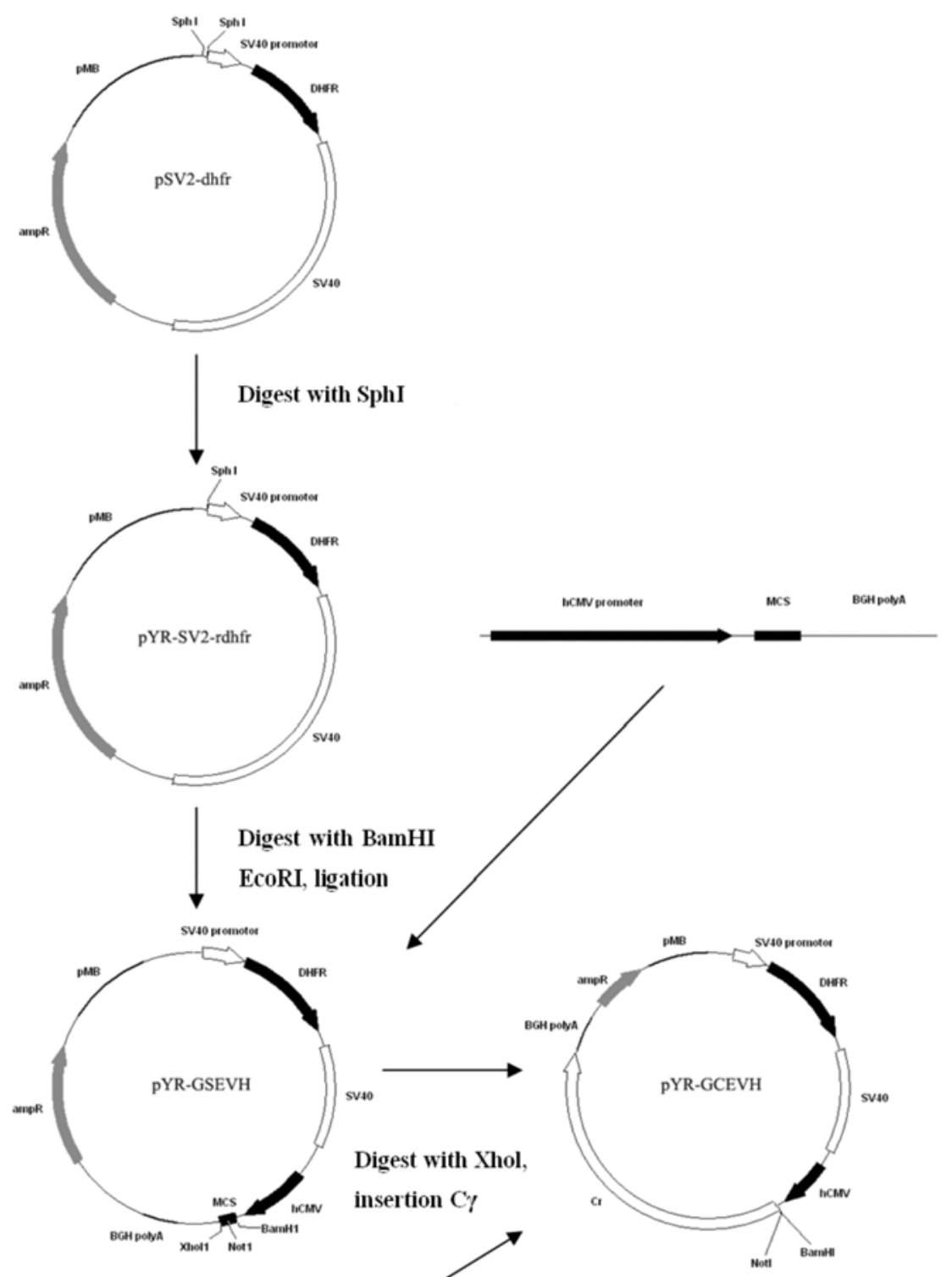

$\mathrm{C} \gamma$

Fig. 1. Schematic diagram of construction of the heavy chain expression vector pYR-GCEVH.

pYR-GCEVL by LipofectAMINE. Stable transfectants were selected by culturing the cells in GHT free DMEM medium with $200 \mu \mathrm{g} \mathrm{ml}^{-1}$ of $\mathrm{G} 418$. Secretion of the chimeric antibody $\mathrm{HCAb}$ was assayed by ELISA. The production was over $1 \mu \mathrm{g}$ $\mathrm{ml}^{-1}$ before amplification.

Methotrexate amplification Significant improvement of expression level in $\mathrm{CHO}$ cells was achieved with vectors amplification. The amplified region is usually larger than that occupied by the marker gene, which resulted in the coamplification of associated sequences including the gene encoding the product (Liu et al., 2004). The target gene was cloned into the multiple cloning sites with transcription initiated by the human cytomegalovirus promoter sequence, while transcription of the DHFR gene was triggered by a weakened SV40 promoter. In order to highly express recombinant antibodies in $\mathrm{CHO}$ cells, the specific DHFR inhibitor MTX was added to pools of transfected cells at concentrations between $3 \times 10^{-8}$ and $10^{-6} \mathrm{M}$. We found that the DHFR-selection system usually produced relatively highlevel expression of chimeric antibodies after only a single round of gene amplification. The average productivity of selected clones at $3 \times 10^{-8} \mathrm{M}$ MTX was over $30 \mu \mathrm{g} \mathrm{ml}^{-1}$, with the highest production of $100 \mu \mathrm{g} \mathrm{ml}^{-1}$ of $\mathrm{HCAb}$ at $10^{-6} \mathrm{M}$ MTX.

Western blot analysis of recombinant Ig molecules Two 

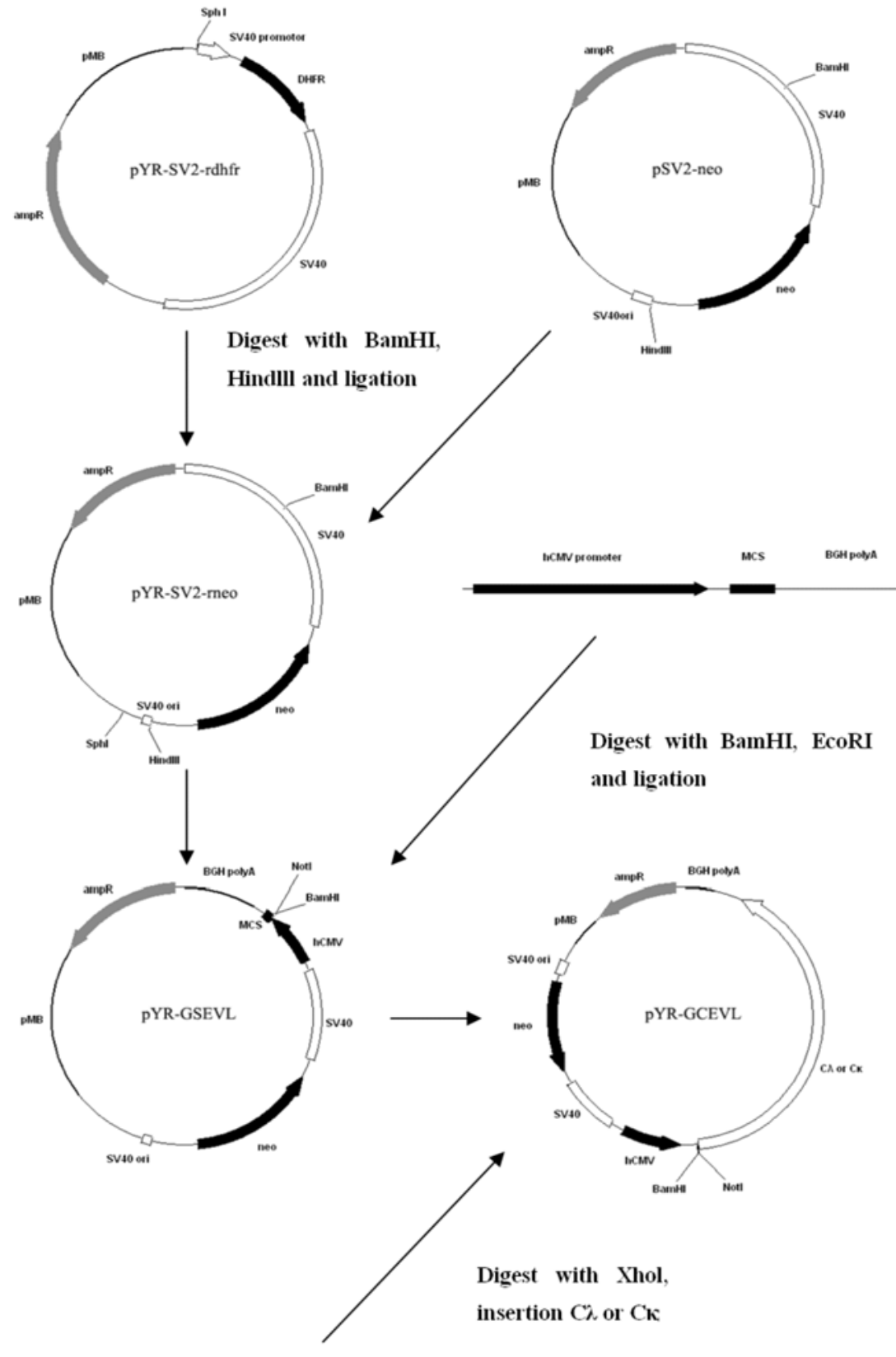

$\mathrm{C} \lambda$ or $\mathrm{Ck}$

Fig. 2. Schematic diagram of construction of the light chain expression vector pYR-GCEVL.

additional analyses were performed to ensure the correct structure and size of the recombinant antibodies. Firstly, an ELISA indicates the secretion of the fully assembled $\mathrm{H}$ chain and L chain from transfected cells. Secondly, western blot was performed to determine the molecular weight of recombinant Ig chain (Fig. 3). Fig. 3 shows that the the size of Ig H chains are approx. $50 \mathrm{kDa}$ as expected, with the size of $\mathrm{Ig} \mathrm{L}$ chains approx. $21 \mathrm{kDa}$. These results, together with the ELISA data, confirmed the correct structure of the individual Ig chains, thus demonstrated the correct polypeptide assembly of the heavy and light chains in the production of the recombinant Ig molecules.

\section{Discussion}

The production of Ig molecules with desired specificities has become an important area of medical and basic research (Mclean et al., 2000). Currently, the process of generating cell lines that produce high levels of recombinant antibody 


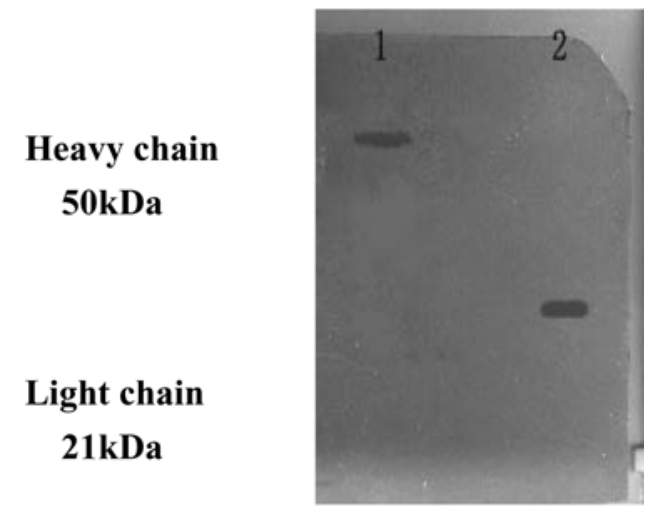

Fig. 3. Western blot analysis of purified chimeric antibody HCAb. Recombinant IgG $(20 \mu 1 /$ lane $)$ purified from culture supernatants of the transfected cells were subjected to SDSPAGE analysis under reducing conditions. Lane 1, the band of human $\gamma$ chain $(50 \mathrm{kDa})$. Lane 2 , the band of human $\kappa$ chain $(21 \mathrm{kDa})$.

requires labor-intensive cloning and screening steps (Kim et al., 2001). This hinders the potential to screen numerous antibody therapeutic candidates and to manufacture them in a timely and cost-effective manner. A system able to rapidly produce high levels of recombinant antibody is needed to relieve this bottlenecked situation (Bianchi et al., 2003).

To express genetically engineered $\mathrm{Ig}$ in $\mathrm{CHO}$ cells, two systems have been frequently utilized, one of which is a twovector system with each Ig chain on a separate plasmid (Wood et al., 1990; Page et al., 1991; Fouser et al., 1992; Tada et al., 1994), the other being the one-vector system with both Ig chains on the same plasmid (Page et al., 1991; Newman et al., 1992; Tada et al., 1994). In our study, the two-vector system was adopted because the heavy and light chain coding sequences could be more easily inserted into the two vectors. Furthermore, with careful designing and construction, the vectors used in this study had already possessed the following characteristics: (a) they can easily express any human-mouse chimeric antibodies by exchanging their V regions; (b) they can both transiently and stably express antibodies; (c) they can express the variable regions of antibodies in different immunoglobulin isotypes.

The expression vectors in this study have been optimized to maximize the production of recombinant product and to minimize the amount of DHFR to allow selection of stable clones. It is generally accepted that DHFR gene can be amplified 500 to 2000 folds in dhfr $^{-}$CHO cells, which consequently leads to the 500- to 2000- fold amplification of the colinked target gene, and finally the several-hundred-fold amplification of the productivity of recombinant protein. Since the target genes are always present at the telomeric ends of amplified arrays in the chromosomes after long-term culture in the absence of MTX, the transfected cells have better hereditary stability (Kim et al., 1999). This mechanism inspired us to use the weakened DHFR gene amplification procedure to increase the recombinant proteins production in lower MTX level.

We chose initial transcription of the immunoglobulin gene from hCMV promoter to facilitate strong expression in mammalian cells, with the optimized peptide, $\mathrm{NH}_{3}$-Met-TyrPhe-Ser-Ala-Ile-Val-Ser-Ala-Ser-Leu-Ser-COOH as a signal. The incorporated SV40 ori enabled the high episomal replication in COS cells and hence the transient expression. Most vectors are generally believed to achieve either transient expression or stable expression. However, the vectors in our study, pYR-GCEVH and pYR-GCEVL, were found not only to express antibodies transiently within 2 days after transfection, permitting preliminary testing of the product, but also to stably express the products for the long term extensive testing. Furthermore, the vectors were discovered to be able to express different isotypes of $\mathrm{Ig}$ because they contain restriction sites facilitating the exchange of the $\mathrm{C}$ regions. Any antibody isotype may be combined with the $\mathrm{V}$ region chosen by substituting the genomic Cã fragment with other genomic constant region.

Another advantage of using two vectors in this study is the presence of the double selection markers (DHFR and Neo). This double selection marker expression system has two merits. First, the two selection markers, present at the heavyand light-chain expression vectors respectively, may balance expression of two chains of antibodies. Second, selecting with two markers may help to delete the pseudo-clones. However, due to the lack of detailed knowledge in the events leading to the integration of nonhomologous plasmids into chromosomal DNA of CHO cells (Kim, 1998), the disproportionate amplification and/or expression of Ig chains may be a drawback in using a two-vector system (Trill, 1995) for it is difficult to control the proportional amplification and/or expression of each Ig chain. In order to overcome this drawback, we attempted to express more heavy chains by including the weakened DHFR gene in the heavy chain expression vector to balance the expression of the heavy and light chains because most IgG-producing mouse myeloma tumors and cell lines are known to synthesize more light chains than heavy chains. Furthermore, the cells with proportional amplification and/or expression of Ig chains can be obtained by extensive screening. To achieve this aim, the constructed expression vectors in our study were cotransfected into DHFR-deficient CHO cells. After initial selection, the parental clone was subjected to DHFR-mediated gene amplification in medium containing stepwise increments in MTX followed by limiting dilution cloning to identify the highest producer subclones at each MTX level.

In this study, the antibody output of the most productive subclone producer was found to be rapidly increased when MTX level rose. We tested the usefulness of the vectors by constructing human-mouse chimeric antibody-HCAb (recently found in our laboratory). After 3 rounds of screening, the secretion of recombinant chimeric antibody-HCAb was found to reach over $100 \mathrm{mg} \mathrm{L}^{-1}$ (30 $\mathrm{pg}$ cell $^{-1}$ day $^{-1}$ ) at $10^{-6} \mathrm{M}$ MTX. The Ig chains were also demonstrated to retain correct $\mathrm{H}$ and 
L chain assembly by western blot analysis. These two findings indicated that the vectors highly expressing chimeric monoclonal antibodies had been successfully constructed, which would consequently facilitate the production of functional recombinant Ig with exchanged $\mathrm{V}$ regions.

In conclusion, we present here a study of the mammalian expression system that is less time-consuming and more costeffective in the mass production of full-length antibody molecules in stable pools. Unlike most previously described antibody expression systems, our vectors have been proved to have the following characteristics: (a) they can easily express any human-mouse chimeric antibodies by exchanging their $\mathrm{V}$ regions; (b) they can both transiently and stably express antibodies in eukaryotic cells; (c) they can express the variable regions of antibodies in different immunoglobulin isotypes; (d) they can balance the expression of two chains of antibodies; and (e) they can enable the effective amplification and the mass production of recombinant antibodies, providing a possible solution to the bottlenecked recombinant antibody expression.

Acknowledgments This research is supported by Chinese Hi-Tech Project (863 Plan) (2002AA217011). Special thanks go to Ms. Fan Peng for her careful reading and editing. We thank many colleagues who have donated plasmids for the construction of these vectors.

\section{References}

Burton, D. R. (1991) Human and mouse monoclonal antibodies by repertoire cloning. Trends Biotechnol. 9, 169-175.

Bianchi, A. A. and McGrew, J. T. (2003) High-level expression of full-length antibodies using trans-complementing expression vectors. Biotechnol. Bioeng. 84, 439-444.

Fouser, L. A., Swanberg, S. L., Lin, B. Y., Benedict, M., Kelleher K., Cumming D. A. and Riedel, G. E. (1992) High level expression of a chimeric anti-ganglioside GD2 antibody: Genomic $\kappa$ sequences improve expression in $\mathrm{COS}$ and $\mathrm{CHO}$ cells. Bio/Technology 10, 1121-1127.

Gorman, S. D., Clark, M. R., Routledge, E. G., Cobbold, S. P. and Waldmann, H. (1991) Reshaping a therapeutic CD4 antibody. Proc. Natl. Acad. Sci. USA 88, 4181-4185.

Harris, W. J. and Emery, S. (1993) Therapeutic antibodies: the coming of age. Trends Biotechnol. 11, 42-44.

Kaufman, R. J., Sharp, P. A. and Latt, S. A. (1983) Evolution of chromosomal regions containing transfected and amplified dihydrofolate reductase sequences. Mol. Cell. Biol 3, 699-711.

Kim, N. S., Byun, T. H. and Lee, G. M. (2001) Key determinants in the occurrence of clonal variation in humanized antibody expression of $\mathrm{CHO}$ cells during dihydrofolate reductase mediated gene amplification. Biotechnol Prog. 17, 69-75.

Kim, S. J. (1998) Characterization of chimeric antibody producing $\mathrm{CHO}$ cells in the course of dihydrofolate reductase-mediated gene amplification and their stability in the absence of selective pressure. Biotechnol. Bioeng. 58, 73-84.

Kim, S. J. and Lee, G. M. (1999) Cytogenetic analysis of chimeric antibody-producing $\mathrm{CHO}$ cells in the course of dihydrofolate reductase-mediated gene amplification and their stability in the absence of selective pressure. Biotechnol. Bioeng. 64, 741-749.

Laemmli, U. K. (1970) Cleavage of structural proteins during the assembly of the head of bacteriophage T4. Nature 227, 680685.

Liu, A. Y., Robinson, R. R., Hellstrom, K. E., Murray, E. D., Chang, C. P. and Hellstrom, I. (1987) Chimeric mouse-human IgG1 antibody that can mediate lysis of cancer cells. Proc. Natl. Acad. Sci. USA 84, 3439-3443.

Liu, W. T. and Wei, H. M. (2004) A balanced expression of two chains of heterodimer protein, the human interleukin-12, improves high-level expression of the protein in $\mathrm{CHO}$ cells. Biochem. Biophys. Res. Commun. 313, 287-293.

Mclean, G. R. and Antonio, N. (2000) Human and murine immunoglobulin expression vector cassettes. Mol. Immunol. 37, 837-845.

Morrison, S. L. (1985) Transfectomas provide novel chimeric antibodies. Science 229, 1202.

Newman, R., Alberts, J., Anderson, D., Carner, K., Heard, C., Norton, F., Raab, R., Reef, M., Shuey, S. and Hanna N. (1992) Primatization of recombinant antibodies for immunotherapy of human diseases: A macaque/human chimeric against human CD4. Bio/Technology 10, 1455-1460.

Page, M. J. and Sydenham, M. A. (1991) High level expression of the humanized monoclonal antibody Campath-1H in Chinese hamster ovary cells. Bio/Technology 9, 64-68.

Persic, L., Roberts A., Wliton J., Cattaneo, A., Bradbury, A. and Hoogenboom, H. R. (1997) An integrated vector system for the eukaryotic expression of antibodies or their fragments after selection from phage display libraries. Gene 187, 9-18.

Tada, H., Kurokawa, T., Seita, T., Watanabe, T. and Iwasa, S. (1994) Expression and characterization of a chimeric bispecific antibody against fibrin and against urokinase-type plasminogen activator. J. Biotechnol. 33, 157-174.

Trill, J. J. (1995) Production of monoclonal antibodies in COS and CHO cells. Curr. Opin. Biotechnol. 6, 553-560.

Waldmann, T. A. (1991) Monoclonal antibodies in diagnosis and therapy. Science 252, 1657-1662.

Wood, C. R., Dorner, A. J., Morris, G. E., Alderman, E. M., Wilson, D., O'hara, R. M. and Kaufman, R. J. (1990) High level synthesis of immunoglobulins in Chinese hamster ovary cells. J. Immunol. 145, 3011-3016.

Yang, X. M., Xing, J. L., Yao, X. Y., Zhang, S. H. and Chen, Z. N. (2004) The cloning and expression of Fab gene of MAb CAb-1 against human colorectal cancer. J. Fourth Military Med. Univ. 25, 1768-1771. 